\title{
Application of Adomian Decomposition Method to Bounded and Unbounded Stokes' Problems
}

\author{
Chi-Min Liu $\mathbb{D}^{1}$ and Ray-Yeng Yang ${ }^{2}$ \\ ${ }^{1}$ Division of Mathematics, General Education Center, Chienkuo Technology University, Taiwan \\ ${ }^{2}$ Department of Hydraulic and Ocean Engineering, National Cheng Kung University, Taiwan
}

Correspondence should be addressed to Chi-Min Liu; cmliu@ctu.edu.tw

Received 30 September 2018; Accepted 18 November 2018; Published 28 November 2018

Academic Editor: Richard I. Avery

Copyright (C) 2018 Chi-Min Liu and Ray-Yeng Yang. This is an open access article distributed under the Creative Commons Attribution License, which permits unrestricted use, distribution, and reproduction in any medium, provided the original work is properly cited.

\begin{abstract}
The well-known Stokes' problems are reexamined by applying the Adomian decomposition method (ADM) associated with other mathematical techniques in this paper. Both the finite-depth (bounded) and infinite-depth (unbounded) cases are analyzed. The present paper raises and deals with two major concerns. The first one is that, for Stokes problems, it lacks one boundary condition at the expansion point to fully determine all coefficients of the ADM solution in which an unknown function appears. This unknown function which is dependent on the transformed variable will be determined by the boundary condition at the far end. The second concern is that the derived solution begins to deviate from the exact solution as the spatial variable grows for the unbounded problems. This can be greatly improved by introducing the Padé approximant to satisfy the boundary condition at the far end. For the second problems, the derived ADM solution can be easily separated into the steady-state and the transient parts for a deeper comprehension of the flow. The present result shows an excellent agreement with the exact solution. The ADM is therefore verified to be a reliable mathematical method to analyze Stokes' problems of finite and infinite depths.
\end{abstract}

\section{Introduction}

The Adomian decomposition method (ADM) has been extensively applied to pursue approximate solutions of mathematical as well as practical problems in many disciplines [1]. Without using linearization, perturbation, closure approximation, or discretization methods, the ADM provides an efficient way to study a rather wide class of nonlinear as well as stochastic equations [2]. It can be applied to study ordinary differential equations (ODEs) and partial differential equations (PDEs) for various kinds of problems. The core idea of the ADM is to decompose the target variable(s) or unknown(s) into an infinite series with no requirement of assuming small parameters. In general, the lowest term of the ADM solution is determined by the imposed initial or boundary condition of the problem, and then other higher terms can be calculated by applying the integral operator of recursion form with the help of lower terms.

Among the ADM studies, different aspects and emphases were demonstrated in literature. Herein we briefly review two focal points. The first one is whether the required boundary/initial condition(s) for deriving the ADM solution is fully known or not. For a differential equation, if the highest-order derivative of the unknown function is $n$, one requires $(n-1)$ boundary/initial condition(s) at the same point to obtain the solution with all coefficients determined. Cases with fully known boundary/initial conditions include the studies of $\mathrm{KdV}$ and $\mathrm{mKdV}$ equations [3], Schrödinger equations [4], Boussinesq and KP equations [5], Burgers' and Boussinesq equations [6], the coupled Burgers' equation [7], heat equation [8], and diffusion equation [9]. In contrast to the above problems with fully given conditions, past studies, including the problems of boundary-layer equation [10], Lane-Emden equations [11], and nonlinear Blasius equation and heat transfer equation [12], are lacking one boundary/initial condition to determine all coefficients of the ADM solution. This has to be solved by considering another boundary condition at different point. The second point we concern is the domain range of the problems. For problems in a finite domain, past studies have verified the 
excellent accuracy and reliability of the ADM [9, 11]. As for the infinite/unbounded cases, the Pade approximant is usually applied to improve the accuracy of the ADM at the far end $[3,6,7,10-14]$. Even if the Padé approximant is successful in extending the valid range of the ADM solution, an unbounded problem still increases the difficulty to keep the accuracy in the whole domain. The above concerns are crucial issues discussed in present study.

In fluid mechanics, Stokes' problem [15] may stand for the most important and classical one for viscous flows. It describes a viscous flow which is suddenly driven by either a moving plate with constant speed (the first problem) or an oscillating plate (the second problem). Stokes' problems have been extensively studied by not only mathematical methods [16] but also numerical simulations [17]. Recently, the applicability of the ADM to Stokes' problems was examined in several articles. The ADM was applied to study the steady-state oscillating solution of unbounded Stokes' second problem [18]. In this work, two boundary conditions, the Dirichlet and Neumann conditions at the oscillating plate, are provided to determine the lowest two terms of the solution. Though the Dirichlet boundary condition is prescribed, the Neumann one, however, which cannot be determined by the original PDE system, is given by the exact solution. Accordingly, the applicability of the ADM may not be well verified in this case. Later, the ADM was again used to verify the steady-state oscillating solution of bounded Stokes' second problem [19]. In the above two studies, only the steady-state oscillating solutions are examined which implies the initial condition is not considered or given in the original PDEs. In addition to Stokes' problems, three classical viscous flows in a finite domain, which include the Couette flow, the Poiseuille flow, and the Couette-Poiseuille flow, were analyzed to obtain the steady-state oscillating solutions [20].

To improve the knowledge and verify the accuracy and applicability of the ADM for Stokes' problems, the exact solution, which contains the steady-state and transient parts, will be examined herein by applying the ADM and other mathematical techniques for bounded and unbounded cases. Different from many of past studies, two boundary conditions which are given at different spatial positions, one at $y=0$ and another at $y=h$ (for bounded problems) or $y \longrightarrow$ $\infty$ (for unbounded problems), result in extra mathematical efforts to determine all coefficients of the ADM solution as two conditions are required at $y=0$ for solving the present second-order PDEs. Accordingly, an unknown function appeared in some coefficients of the ADM solution which has to be determined by the boundary condition at $y=h$ or $y \longrightarrow \infty$. For the unbounded case, the Padé approximant is introduced to avoid divergent behaviors when $y$ grows. The organization of present paper is as what follows. In Section 2, the bounded Stokes' problems are examined using the ADM. For the unbounded problems, the ADM as well as the Padé approximants are applied to perform the analysis in Section 3. For all problems, the Laplace transform is required to implement the analysis due to the prescribed initial condition. To verify the accuracy and applicability of the ADM, present solutions are compared with the exact solutions of Stokes' problems. Concluding remarks are made in Section 4.

\section{Bounded Stokes' Problems}

In this section, the bounded (finite-depth) Stokes' first and second problems are analyzed by using the ADM. A Newtonian fluid of dynamic viscosity $v$ is considered occupy the upper $y$ domain with a finite depth $h$. The only driven force comes from the plate located $y=0$ which it begins to either move in a constant speed (the first problem) or oscillate with frequency $\omega$ (the second problem). Plate motion is only allowed in the $x$ direction for $t>0$. Detailed derivations for the two problems are addressed in the following subsections.

2.1. The First Problem. For the first problem, the momentum equation for velocity $u(y, t)$ in $x$ direction, boundary and initial conditions are shown in dimensionless forms

$$
\begin{aligned}
\frac{\partial u}{\partial t} & =\frac{\partial^{2} u}{\partial y^{2}}, \\
u & =1 \quad \text { at } y=0 \text { for } t>0, \\
\frac{\partial u}{\partial y} & =0 \quad \text { at } y=1 \text { for } t>0, \\
u & =0 \quad \text { for } t \leq 0,
\end{aligned}
$$

where the relations with dimensional variables are $y=y^{*} / h$, $t=v t^{*} / h^{2}, u=u^{*} / u_{0}$ and $u_{0}$ is the plate speed. Boundary conditions (2) and (3), respectively, address the plate motion at $y=0$ and the stress-free condition at the free surface $y=1$. Now the Laplace transform defined as

$$
\widehat{u}(s)=\int_{0}^{\infty} u(t) e^{-s t} d t,
$$

is applied to (1) to (3) with the help of (4). The results are

$$
\begin{aligned}
s \widehat{u} & =\frac{\partial^{2} \widehat{u}}{\partial y^{2}}, \\
\widehat{u} & =\frac{1}{s}, \quad \text { at } y=0, \\
\frac{\partial \widehat{u}}{\partial y} & =0, \quad \text { at } y=1 .
\end{aligned}
$$

To begin the ADM analysis [1,2], a linear differential operator and its inverse integral are defined

$$
\begin{aligned}
\mathrm{L}_{y}(.) & =\frac{\partial^{2}}{\partial y^{2}}(.), \\
\mathrm{L}_{y}^{-1}(.) & =\int_{0}^{y} \int_{0}^{y}(.) d y d y .
\end{aligned}
$$

Now we apply (10) into (6) and then solve it by applying the boundary condition at $y=0$. It yields

$$
\widehat{u}(y, s)=\frac{1}{s}+\widehat{g}(s) \cdot y+\int_{0}^{y} \int_{0}^{y} s \widehat{u} d y d y .
$$


In (11), the unknown $\hat{g}(s)$ stands for the Neumann boundary condition at $y=0$, i.e., $u_{y}(y=0)$. Different from many studies in which the unknown is a constant, $\widehat{g}(s)$ is dependent on the transformed variable $s$. Values of $\widehat{g}(s)$ will be determined later. Now we assume the transformed velocity $\widehat{u}$ to be of a series form

$$
\widehat{u}(y, s)=\sum_{i=1}^{\infty} \widehat{u}_{i}(y, s) .
$$

The first-order term of (12) is assigned by using the boundary conditions at $y=0$,

$$
\widehat{u}_{1}=\frac{1}{s}+\widehat{g}(\mathrm{~s}) y,
$$

and higher-order terms are determined by a recursion relation

$$
\widehat{u}_{n+1}=\int_{0}^{y} \int_{0}^{y} s \widehat{u}_{n} d y d y \text { for } n \geq 1 .
$$

After some algebra, higher-order terms are solved:

$$
\begin{aligned}
& \widehat{u}_{2}=\frac{1}{2} y^{2}+\frac{s \widehat{g}(s)}{6} y^{3}, \\
& \widehat{u}_{3}=\frac{s}{24} y^{4}+\frac{s^{2} \widehat{g}(s)}{120} y^{5}, \\
& \widehat{u}_{4}=\frac{s^{2}}{720} y^{6}+\frac{s^{3} \widehat{g}(s)}{5040} y^{7}, \\
& \widehat{u}_{n}=\frac{s^{n-2}}{(2 n-2) !} y^{2 n-2}+\frac{s^{n-1} \widehat{g}(s)}{(2 n-1) !} y^{2 n-1} .
\end{aligned}
$$

Now we define the approximate transformed solution of order $n$ is denoted by $\widehat{U}_{n}$ :

$$
\widehat{U}_{n}(y, s)=\sum_{i=1}^{n} \widehat{u}_{i}(y, s) .
$$

As all approximate transformed solutions $\widehat{U}_{n}$ contain the unknown $\widehat{g}(s)$, it implies that the determination of value of $\widehat{g}(s)$ will differ and depend on the approximate order $n$. The unknown $\widehat{g}(s)$ is now determined by making $\widehat{U}_{n}$ to satisfy the boundary condition at the free surface at $y=1$ :

$$
\frac{\partial \widehat{U}_{n}}{\partial y}=0 \quad \text { at } y=1 .
$$

The results for $\widehat{U}_{1}$ to $\widehat{U}_{4}$ are

$$
\begin{aligned}
& \widehat{g}_{1}(s)=0 \text { for } \widehat{U}_{1}, \\
& \widehat{g}_{2}(s)=\frac{-2}{s+2} \text { for } \widehat{U}_{2}, \\
& \widehat{g}_{3}(s)=\frac{-4(s+6)}{s^{2}+12 s+24} \text { for } \widehat{U}_{3}, \\
& \widehat{g}_{4}(s)=\frac{-6\left(s^{2}+20 s+120\right)}{s^{3}+30 s^{2}+360 s+720} \text { for } \widehat{U}_{4} .
\end{aligned}
$$

By calculating the inverse Laplace transform of (19) with the help of (21) to (24), one now can obtain the approximate solutions $U_{n}$ :

$$
\begin{aligned}
U_{1}= & 1, \\
U_{2}= & 1+\frac{2 y^{3}-6 y}{3} \cdot e^{-2 t}+\frac{-2 y^{3}+3 y^{2}}{6} \cdot \delta(t), \\
U_{3}= & 1+\frac{6 y^{5}-20 y^{3}+15 y^{2}}{30} \cdot \delta(t)+\frac{-4 y^{5}+5 y^{4}}{120} \\
& \cdot \delta^{\prime}(\mathrm{t}) \\
& -\frac{2 y\left(15-5(3+\sqrt{3}) y^{2}+3(2+\sqrt{3}) y^{4}\right)}{15} \\
& \cdot e^{-2(3+\sqrt{3}) t} \\
& -\frac{2 y\left(15-5(3-\sqrt{3}) y^{2}+3(2-\sqrt{3}) y^{4}\right)}{15} \\
& \cdot e^{-2(3-\sqrt{3}) t},
\end{aligned}
$$

where $\delta(\bullet)$ represent the Dirac Delta function, and $U_{4}$ is ignored herein due to its very length expression. For measuring the accuracy of ADM solutions, the exact solution used for comparison is [21]

$$
\begin{aligned}
U_{\text {exact }}= & 1-\frac{4}{\pi} \sum_{n=0}^{\infty} \frac{1}{2 n+1} \\
& \cdot \exp \left[-\frac{(2 n+1)^{2} \pi^{2}}{4} t\right] \sin \left[\frac{(2 n+1) \pi}{2} y\right] .
\end{aligned}
$$

Figures 1(a) and 1(b) show the velocity profiles of $U_{1}$ to $U_{4}$ by dash curves of different colors and $U_{\text {exact }}$ by solid curve for the cases of $t=0.5$ and $t=1$, respectively. For this bounded problem, it is found that a higher-order ADM solution approaches the exact solution much closer than a lower-order one. For quantitatively comparing the accuracy of the approximate solution $U_{n}$ with the exact solution, a parameter

$$
E_{n}=\left|\frac{U_{n}-U_{\text {exact }}}{U_{\text {exact }}}\right|
$$

is defined to measure the difference between the exact and ADM solutions. Table 1 shows the error percentages at the free surface $y=1$ where the maximum error may occur for each approximate solution. Results show that the difference decreases as time grows. It is remarked that, as $U_{1}=1$ is equivalent to the speed of moving plate, all other approximate solutions will gradually approach to $U_{1}$ as time approaches infinity. 
TABLE 1: Error percentages $E_{n}$ at $y=1$.

\begin{tabular}{lcccc}
\hline & $E_{1}$ & $E_{2}$ & $E_{3}$ & \\
\hline$t=0.5$ & $58.93 \%$ & $19.03 \%$ & $2.02 \%$ & $0.24 \%$ \\
$t=1$ & $12.10 \%$ & $8.12 \%$ & $0.90 \%$ & $0.04 \%$ \\
\hline
\end{tabular}
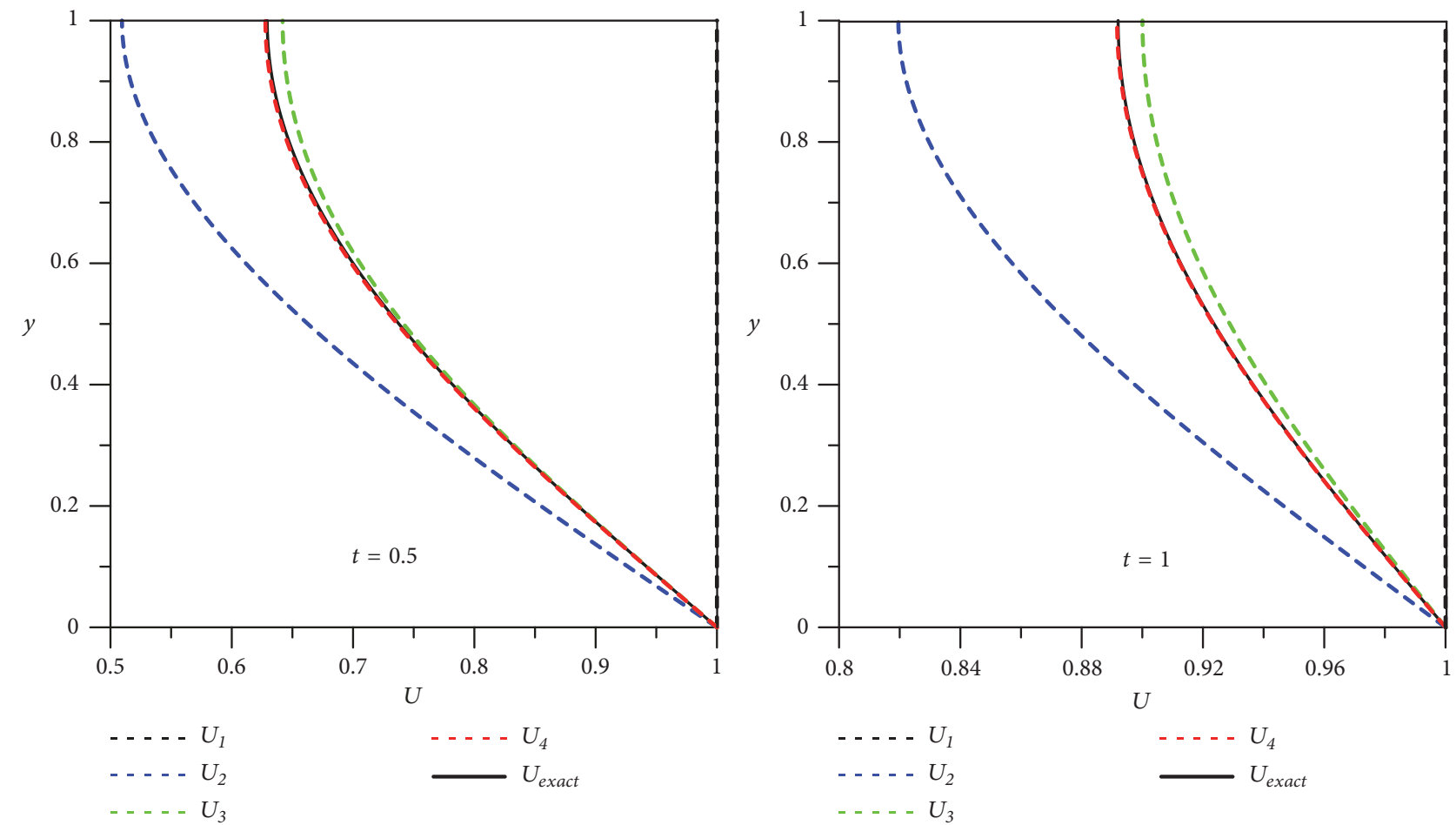

FIGURE 1: Velocity profiles of the exact and ADM solutions for the bounded Stokes' first problem. (a) $t=0.5$. (b) $t=1$.

2.2. The Second Problem. For the second problem, the governing equation, boundary and initial conditions are

$$
\begin{aligned}
\frac{\partial u}{\partial t} & =\lambda \frac{\partial^{2} u}{\partial y^{2}}, \\
u & =\cos t \quad \text { at } y=0 \text { for } t>0, \\
\frac{\partial u}{\partial y} & =0 \quad \text { at } y=1 \text { for } t>0, \\
u & =0 \quad \text { for } t \leq 0,
\end{aligned}
$$

where the relations with dimensional variables are $y=y^{*} / h$, $t=\omega t^{*}, u=u^{*} / u_{0}$, and $\lambda=v / \omega h^{2}$. The only difference between the first and second problems is the boundary condition at the plate addressed by (2) and (31). For the reason of simplicity and without losing generality of the problem, the value of $\lambda$ which indicates the viscous effects is assigned to be unity henceforth. By following the same procedures presented in previous subsection, the transformed solution is shown as

$$
\widehat{u}_{1}=\frac{s}{s^{2}+1}+\widehat{g}(\mathrm{~s}) y
$$

$$
\begin{aligned}
& \widehat{u}_{2}=\frac{s}{2}\left(\frac{s}{s^{2}+1} y^{2}+\frac{\widehat{g}(s)}{3} y^{3}\right), \\
& \widehat{u}_{3}=\frac{s^{2}}{24}\left(\frac{s}{s^{2}+1} y^{4}+\frac{\widehat{g}(s)}{5} y^{5}\right), \\
& \widehat{u}_{n}=\frac{s^{n-1}}{(2 n-2) !}\left(\frac{s}{s^{2}+1} y^{2 n-2}+\frac{\widehat{g}(s)}{2 n-1} y^{2 n-1}\right) .
\end{aligned}
$$

The unknown $\widehat{g}_{n}$ for each approximate solution is solved by letting it for the transformed approximate solutions $\widehat{U}_{1}$ to $\widehat{U}_{4}$, as defined in (19), satisfy the boundary condition at $y=1$. It reads

$$
\begin{aligned}
& \widehat{g}_{1}(s)=0 \text { for } \widehat{U}_{1}, \\
& \widehat{g}_{2}(s)=\frac{-2 s^{2}}{\left(s^{2}+1\right)(s+2)} \text { for } \widehat{U}_{2}, \\
& \widehat{g}_{3}(s)=\frac{-4 s^{2}(s+6)}{\left(s^{2}+1\right)\left(s^{2}+12 s+24\right)} \text { for } \widehat{U}_{3}, \\
& \widehat{g}_{4}(s)=\frac{-6 s^{2}\left(s^{2}+20 s+120\right)}{\left(s^{2}+1\right)\left(s^{3}+30 s^{2}+360 s+720\right)} \text { for } \widehat{U}_{4}
\end{aligned}
$$


TABLE 2: Error percentages $E_{n}$ at $y=1$.

\begin{tabular}{lcccc}
\hline & $E_{1}$ & $E_{2}$ & $E_{3}$ & $E_{4}$ \\
\hline$t=\pi$ & $21.49 \%$ & $10.67 \%$ & $0.56 \%$ & $0.16 \%$ \\
$t=2 \pi$ & $21.55 \%$ & $10.86 \%$ & $0.55 \%$ & $0.17 \%$ \\
\hline
\end{tabular}

The approximate solutions $U_{1}$ to $U_{4}$ can be obtained by performing the inverse Laplace transform to $\widehat{U}_{1}$ to $\widehat{U}_{4}$. The results are

$$
\begin{aligned}
U_{1} & =\cos t, \\
U_{2} & =\frac{-2 y^{3}+3 y^{2}}{6} \cdot \delta(t)+\frac{8 y^{3}-24 y}{15} \cdot e^{-2 t}+\frac{2 y^{3}-6 \mathrm{y}+15}{15} \\
& \cdot \cos t+\frac{2 y^{3}-15 y^{2}+24 y}{30} \cdot \sin t, \\
U_{3} & =\frac{6 y^{5}-20 y^{3}+15 y^{2}}{30} \cdot \delta(t)+\frac{4 y^{5}-5 y^{4}}{120} \cdot \delta^{\prime}(\mathrm{t}) \\
& -\frac{16 y\left((165+84 \sqrt{3}) y^{4}-(405+145 \sqrt{3}) y^{2}+390+15 \sqrt{3}\right)}{3365} \\
& \cdot e^{-2(3+\sqrt{3}) t} \\
& -\frac{16 y\left((165-84 \sqrt{3}) y^{4}-(405-145 \sqrt{3}) y^{2}+390-15 \sqrt{3}\right)}{3365} \\
& \cdot e^{-2(3-\sqrt{3}) t}+\frac{196 y^{5}-3365 y^{4}+12000 y^{3}-23520 y+80760}{80760} \\
& \cdot \cos t+\frac{-600 y^{5}+3920 y^{2}-40380 y+72000}{80760} \cdot \sin t .
\end{aligned}
$$

where $U_{4}$ is ignored again due to its lengthy expression. It is found that the approximate solutions are constituted by oscillating terms, $\cos t$ and $\sin t$, exponential terms, and the Dirac delta function $\delta(t)$ and its derivative. Morever, terms related to $\cos t$ and $\sin t$ constitute the steady-state oscillating solution while exponential terms are the transient components which decay as time grows.

Figure 2 displays the velocity profiles at $t=\pi$ and $t=2 \pi$. The exact solution for comparison is introduced [21]:

$$
\begin{aligned}
U_{\text {exact }}= & \cos t+2 \sum_{i=0}^{\infty} \frac{\sin n y}{n} \\
& \cdot\left(-e^{-n^{2} t}+\frac{n^{2} \sin t-\cos t+e^{-n^{2} t}}{n^{4}+1}\right),
\end{aligned}
$$

where

$$
n=\frac{2 i+1}{2} \pi
$$

It shows that both $U_{3}$ and $U_{4}$ show excellent behaviors in comparison with the exact solution. Table 2 displays the difference ratio between the ADM solution and the exact solution. No obvious difference appears for approximate solutions of all orders at the first round of oscillation.

\section{Unbounded Stokes' Problems}

The unbounded Stokes' problem, which describes the induced viscous flow in an infinite-depth domain, is investigated in this section. In general, a problem in an unbounded domain usually shows divergent behaviors as values of some variables or parameters increase, especially by using methods based on a series expansion. Accordingly, in addition to the $\mathrm{ADM}$, the Padé approximant is introduced to improve the accuracy of velocity profile in the unbounded domain. In the following two subsections, the first and second problems will be examined, respectively. All variables, conditions and physical definitions are the same with those shown in previous section, except the position of the upper boundary is moved form $y=h$ to $y \longrightarrow \infty$.

3.1. The First Problem. For the Stokes' first problem, the momentum equation in $x$ direction, boundary and initial conditions are

$$
\begin{aligned}
& \frac{\partial u}{\partial t}=\frac{\partial^{2} u}{\partial y^{2}}, \\
& u=1 \quad \text { at } y=0 \text { for } t>0, \\
& u=0 \quad \text { at } y \longrightarrow \infty \text { for } t>0, \\
& u=0 \quad \text { for } t \leq 0 .
\end{aligned}
$$

By following the same procedures shown in previous section, the transformed ADM solutions $\widehat{U}_{4}$ is

$$
\begin{aligned}
\widehat{U}_{4}= & \frac{1}{s}+\widehat{g} y+\frac{1}{2} y^{2}+\frac{s \widehat{g}}{6} y^{3}+\frac{s}{24} y^{4}+\frac{s^{2} \widehat{g}}{120} y^{5} \\
& +\frac{s^{2}}{720} y^{6}+\frac{s^{3} \widehat{g}}{5040} y^{7} .
\end{aligned}
$$

It is noted again that the unknown $\widehat{g}(s)$ will be determined by applying the boundary condition at $y \longrightarrow \infty$. As (51) quickly diverges while $y$ goes large, the Padé approximant is now applied to transform (51) into the following Padé forms:

$$
\widehat{U}_{[1,1]}=\frac{1+\left(\left(2 \hat{g}^{2} s-1\right) / 2 \widehat{g}\right) y}{s[1-(1 / 2 \widehat{g}) y]}
$$




$$
\begin{aligned}
& \widehat{U}_{[2,2]}=\frac{1+\left(\left(30 \widehat{g} s-24 \widehat{g}^{3} s^{2}\right) /\left(36-24 \widehat{g}^{2} s\right)\right) y+\left(\left(15 s-14 \widehat{g}^{2} s^{2}\right) /\left(36-24 \widehat{g}^{2} s\right)\right) y^{2}}{s\left[1+\left((-6 \widehat{g} s) /\left(36-24 \widehat{g}^{2} s\right)\right) y+\left(\left(4 \widehat{g}^{2} s^{2}-3 s\right) /\left(36-24 \widehat{g}^{2} s\right)\right) y^{2}\right]}, \\
& \widehat{U}_{[3,3]}=\left(1+\frac{\left(-540+3840 \widehat{g}^{2} s-3360 \widehat{g}^{4} s^{2}\right)}{\left(3240 \widehat{g}-3360 \widehat{g}^{3} s\right)} y+\frac{\left(900 \widehat{g} s-912 \widehat{g}^{3} s^{2}\right)}{\left(3240 \widehat{g}-3360 \widehat{g}^{3} s\right)} y^{2}+\frac{\left(-225 s+616 \widehat{g}^{2} s^{2}-392 \widehat{g}^{4} s^{3}\right)}{\left(3240 \widehat{g}-3360 \widehat{g}^{3} s\right)} y^{3}\right) \\
& \quad \cdot\left(s\left[1+\frac{\left(-540+600 \widehat{g}^{2} s\right)}{\left(3240 \hat{g}-3360 \widehat{g}^{3} s\right)} y+\frac{\left(-180 \widehat{g} s+168 \widehat{g}^{3} s^{2}\right)}{\left(3240 \hat{g}-3360 \widehat{g}^{3} s\right)} y^{2}+\frac{\left(45 s-44 \widehat{g}^{2} s^{2}\right)}{\left(3240 \widehat{g}-3360 \widehat{g}^{3} s\right)} y^{3}\right]\right)^{-1},
\end{aligned}
$$

where the subscript $[m, n]$ denotes the order of the Padé approximant. To satisfy the boundary condition at $y \longrightarrow \infty$, it requires that the highest term of $y$ power in the numerator has to vanish. It reads

$$
\widehat{g}(s)= \begin{cases} \pm \sqrt{\frac{1}{2 s}} & \text { for } \widehat{U}_{[1,1]} \\ \pm \sqrt{\frac{15}{14 s}} & \text { for } \widehat{U}_{[2,2]} \\ \pm \frac{1}{2} \sqrt{\frac{22 \pm \sqrt{34}}{7 s}} & \text { for } \widehat{U}_{[3,3]}\end{cases}
$$

As the derivation of $U_{[3,3]}$ can be readily performed by following the same derivation for $U_{[1,1]}$ and $U_{[2,2]}$, for the sake of a brief and clear demonstration, we only show details of the derivation for $U_{[1,1]}$ and $U_{[2,2]}$ and neglect the display of $U_{[3,3]}$ henceforward. Applying the minus values of $\widehat{g}(s)$ in (55) to (52) and (53) results in

$$
\begin{aligned}
\widehat{U}_{[1,1]} & =\frac{2}{2 s+\sqrt{2} s^{1.5} y}, \\
\widehat{U}_{[2,2]} & =\frac{168-5 \sqrt{210} \sqrt{s} y}{168 s+7 \sqrt{210} s^{1.5} y+21 s^{2} y^{2}} .
\end{aligned}
$$

It is remarked that, if one takes the plus values of $\widehat{g}(s)$ in (55), the derived velocity profiles will fall into an invalid range. $U_{[1,1]}$ and $U_{[2,2]}$ now can be calculated by performing the inverse Laplace transform

$$
U=\frac{1}{2 \pi i} \int_{k+i \infty}^{k-i \infty} \widehat{U} e^{s t} d s
$$

to (56) and (57). The path of the contour integration is shown in Figure 3. Inside the contour it has two poles $s_{1}$ and $s_{2}$ for $U_{[2,2]}$. Therefore, we have

$$
\begin{gathered}
U_{[1,1]}=-\frac{1}{2 \pi i}\left(\int_{A B}+\int_{B C}+\int_{C D E}+\int_{E F}\right. \\
\left.+\int_{F G} \widehat{U}_{[1,1]} e^{s t} d s\right)
\end{gathered}
$$

$$
\begin{aligned}
& U_{[2,2]}=-\frac{1}{2 \pi i}\left(\int_{A B}+\int_{B C}+\int_{C D E}+\int_{E F}\right. \\
& \left.+\int_{F G} \widehat{U}_{[2,2]} e^{s t} d s\right)+\operatorname{Res}\left(\frac{11-\sqrt{455 i}}{3 y^{2}}\right) \\
& +\operatorname{Res}\left(\frac{11+\sqrt{455 i}}{3 y^{2}}\right),
\end{aligned}
$$

where the integrations along the paths $A B$ and $F G$ are zero, the integration along the path $C D E$ is $-2 \pi i$, and $\operatorname{Res}(\bullet)$ represents the residues of poles inside the contour. After some algebra, (59) and (60) are further calculated to be

$$
\begin{aligned}
& U_{[1,1]}=1-\frac{\sqrt{2} y}{\pi} \int_{\alpha=0}^{\infty} \frac{\alpha^{0.5}}{\alpha(\alpha y+2)} \cdot e^{-\alpha t} d \alpha, \\
& U_{[2,2]}=1+\frac{\sqrt{210} y}{21 \pi} \int_{\alpha=0}^{\infty} \frac{5 \alpha y^{2}-96}{\left(\alpha y^{2}+11 / 3\right)^{2}+455 / 9} \\
& . \frac{e^{-\alpha t}}{\sqrt{\alpha}} d \alpha+\frac{\exp \left(11 t / 3 y^{2}\right)}{7 \sqrt{455}}\left[-50 \sqrt{70} r^{0.5}\right. \\
& \cdot \sin \left(\frac{\theta}{2}+\frac{\sqrt{455}}{3 y^{2}} t\right)+518 \sin \left(\frac{\sqrt{455}}{3 y^{2}} t\right) \\
& -\frac{288 \sqrt{70}}{r^{0.5}} \sin \left(-\frac{\theta}{2}+\frac{\sqrt{455}}{3 y^{2}} t\right) \\
& \left.+\frac{4032}{r} \sin \left(-\theta+\frac{\sqrt{455}}{3 y^{2}} t\right)\right],
\end{aligned}
$$

with the relations

$$
\begin{aligned}
& r=24 \\
& \theta=\tan ^{-1} \frac{\sqrt{455}}{11} .
\end{aligned}
$$

The exact solution for the first problem is introduced for comparison [16]:

$$
U_{\text {exact }}=\operatorname{Erfc}\left(\frac{y}{2 \sqrt{t}}\right)
$$

where $\operatorname{Erfc}(\bullet)$ denotes the complementary error function. The ADM-Padé solutions and the exact solution are plotted in Figure 4 for the cases of $t=1$ and $t=5$. For the range $0 \leq y \leq 5, U_{[3,3]}$ has an excellent agreement with the exact solution while behaviors of $U_{[1,1]}$ and $U_{[2,2]}$ are comparatively poor. It is also found that the difference becomes smaller for all approximate solutions at larger times. 

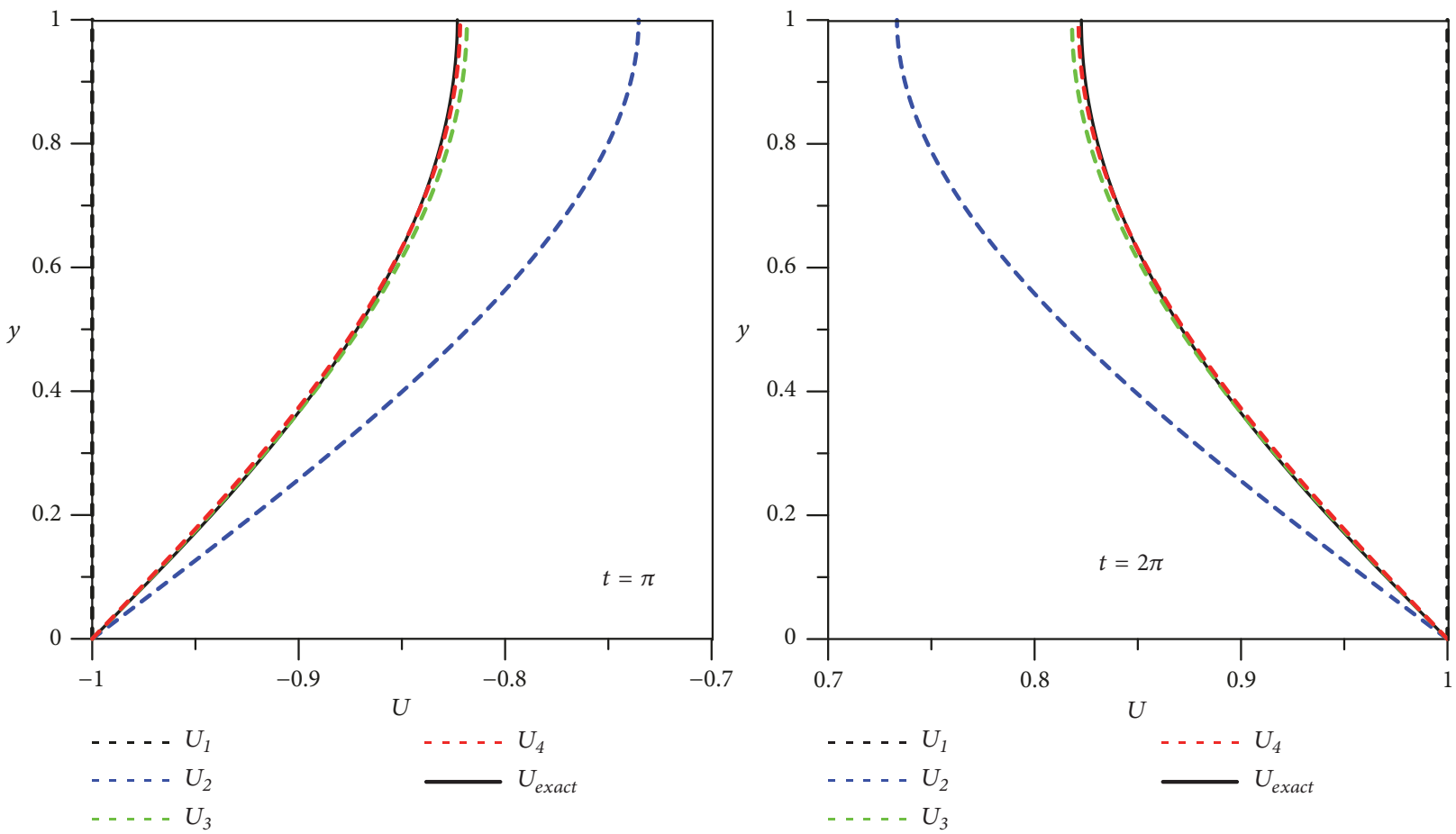

FIGURE 2: Velocity profiles of the exact and ADM solutions for the bounded Stokes' second problem. (a) $t=\pi$. (b) $t=2 \pi$.

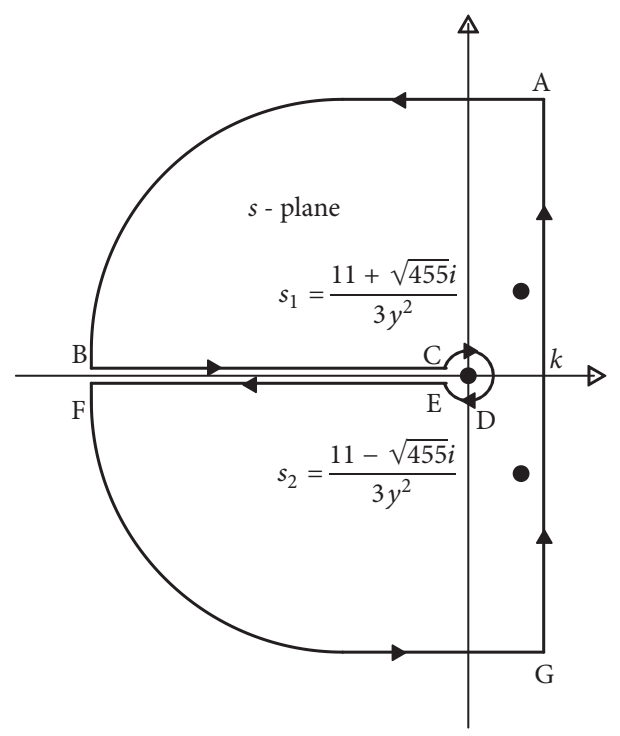

FIgURE 3: Contour integral of unbounded Stokes' first problem. The poles $s_{1}$ and $s_{2}$ are for $U_{[2,2]}$ only.

3.2. The Second Problem. For the second problem of infinite depth, all equations are the same with the finitedepth case except the boundary condition as $y$ approaches infinity:

$$
\begin{aligned}
\frac{\partial u}{\partial t} & =\lambda \frac{\partial^{2} u}{\partial y^{2}}, \\
u & =\cos t \quad \text { at } y=0 \text { for } t>0, \\
u & =0 \quad \text { at } y \longrightarrow \infty \text { for } t>0, \\
u & =0 \quad \text { for } t \leq 0,
\end{aligned}
$$

By letting $\lambda$ be unity and following the same derivation shown previously, the 4 th-order transformed solution is

$$
\begin{aligned}
\widehat{U}_{4}= & \frac{s}{s^{2}+1}+\widehat{g} y+\frac{s^{2}}{2\left(s^{2}+1\right)} y^{2}+\frac{s \widehat{g}}{6} y^{3} \\
& +\frac{s^{3}}{24\left(s^{2}+1\right)} y^{4}+\frac{s^{2} \widehat{g}}{120} y^{5}+\frac{s^{4}}{720\left(s^{2}+1\right)} y^{6} \\
& +\frac{s^{3} \widehat{g}}{5040} y^{7} .
\end{aligned}
$$

Therefore the transformed ADM-Padé approximants are 


$$
\begin{aligned}
& \widehat{U}_{[1,1]}=\frac{s\left[1+\left(\left(s^{2}+1\right) \hat{g} / s-s^{2} / 2\left(s^{2}+1\right) \hat{g}\right) y\right]}{\left(s^{2}+1\right)\left[1-\left(s^{2} / 2\left(s^{2}+1\right) \hat{g}\right) y\right]}, \\
& \widehat{U}_{[2,2]} \\
& =\frac{s\left[1+\left(\left(s^{2}+1\right) \hat{g} / s+\left(\widehat{g} s^{2}\left(s^{2}+1\right) /\left(-6 s^{3}+4 \widehat{g}^{2}\left(s^{2}+1\right)^{2}\right)\right)\right) y+\left(\left(-15 s^{4}+14 \widehat{g}^{2} s\left(s^{2}+1\right)^{2}\right) / 12\left(-3 s^{3}+2 \widehat{g}^{2}\left(s^{2}+1\right)^{2}\right)\right) y^{2}\right]}{\left(s^{2}+1\right)\left[1+\left(\widehat{g} s^{2}\left(s^{2}+1\right) /\left(-6 s^{3}+4 \hat{g}^{2}\left(s^{2}+1\right)^{2}\right)\right) y+\left(\left(3 s^{4}-4 \widehat{g}^{2} s\left(s^{2}+1\right)^{2}\right) / 12\left(-3 s^{3}+2 \widehat{g}^{2}\left(s^{2}+1\right)^{2}\right)\right) y^{2}\right]} \\
& \widehat{U}_{[3,3]}=\left(s \left[1+\left(\frac{\left(s^{2}+1\right) \hat{g}}{s}-\frac{s^{5} / 1920\left(s^{2}+1\right)^{3}+-\widehat{g} s^{2} / 1728\left(s^{2}+1\right)}{7 \widehat{g}^{3} s^{3} / 2160-\widehat{g} s^{6} / 320\left(s^{2}+1\right)^{2}}\right) y+\left(\frac{19 \widehat{g}^{3} s^{5} / 21600\left(s^{2}+1\right)-\widehat{g} s^{8} / 1152\left(s^{2}+1\right)^{3}}{7 \widehat{g}^{3} s^{3} / 2160-\hat{g} s^{6} / 320\left(s^{2}+1\right)^{2}}\right) y^{2}\right.\right. \\
& \left.\left.+\left(\frac{49 \widehat{g}^{4} s^{4} / 129600+s^{10} / 4608\left(s^{2}+1\right)^{4}-77 \widehat{g}^{2} s^{7} / 129600\left(s^{2}+1\right)^{2}}{7 \widehat{g}^{3} s^{3} / 2160-\widehat{g} s^{6} / 320\left(s^{2}+1\right)^{2}}\right) y^{3}\right]\right)\left(\left(s^{2}+1\right)[1\right. \\
& +\left(\frac{s^{8} / 1920\left(s^{2}+1\right)^{3}-\widehat{g}^{2} s^{5} / 1728\left(s^{2}+1\right)}{7 \widehat{g}^{3} s^{3} / 2160-\widehat{g} s^{6} / 320\left(s^{2}+1\right)^{2}}\right) y+\left(\frac{\widehat{g} s^{7} / 5760\left(s^{2}+1\right)^{2}-7 \widehat{g}^{3} s^{4} / 43200}{7 \widehat{g}^{3} s^{3} / 2160-\widehat{g} s^{6} / 320\left(s^{2}+1\right)^{2}}\right) y^{2} \\
& \left.\left.++\left(\frac{11 \widehat{g}^{6} / 259200\left(s^{2}+1\right)-s^{9} / 23040\left(s^{2}+1\right)^{3}}{7 \hat{g}^{3} s^{3} / 2160-\hat{g} s^{6} / 320\left(s^{2}+1\right)^{2}}\right) y^{3}\right]\right)^{-1}
\end{aligned}
$$

The unknown $\widehat{g}(s)$ in above equations is solved by setting the coefficient of the highest $y$ term in the numerator to be zero. It reads

$$
\widehat{g}(s)= \begin{cases} \pm \frac{s^{1.5}}{\sqrt{2}\left(s^{2}+1\right)} & \text { for } \widehat{U}_{[1,1]} \\ \pm \frac{\sqrt{15} s^{1.5}}{\sqrt{14}\left(s^{2}+1\right)} & \text { for } \widehat{U}_{[2,2]} \\ \pm \frac{\sqrt{22 \pm \sqrt{34}} s^{1.5}}{2 \sqrt{7}\left(s^{2}+1\right)} & \text { for } \widehat{U}_{[3,3]}\end{cases}
$$

By applying values of $\widehat{g}(s)$ in (73) into (70) and (71), and then performing the inverse Laplace transform with the help of the contour integral shown in Figure 5, the results are (results for $U_{[3,3]}$ is neglected)

$$
\begin{aligned}
U_{[1,1]}= & -\frac{\sqrt{2} y}{\pi} \int_{\alpha=0}^{\infty} \frac{\alpha^{1.5} e^{-\alpha t}}{\left(\alpha^{2}+1\right)\left(\alpha y^{2}+2\right)} d \alpha+\operatorname{Res}(i) \\
& +\operatorname{Res}(-i),
\end{aligned}
$$

where the summation of two residues are

$$
\frac{1}{y^{4}+4}\left[\left(4-2 y+y^{3}\right) \cos t+\left(2 y-2 y^{2}+y^{3}\right) \sin t\right]
$$

and

$$
\begin{aligned}
U_{[2,2]}= & \frac{\sqrt{210} y}{21 \pi} \int_{\alpha=0}^{\infty} \frac{5 \alpha y^{2}-96}{\left(\alpha y^{2}+11 / 3\right)^{2}+455 / 9} \\
& \cdot \frac{\alpha^{1.5} e^{-\alpha t}}{\alpha^{2}+1} d \alpha+\operatorname{Res}(i)+\operatorname{Res}(-i) \\
& +\operatorname{Res}\left(\frac{11-\sqrt{455} i}{3 y^{2}}\right) \\
& +\operatorname{Res}\left(\frac{11+\sqrt{455} i}{3 y^{2}}\right),
\end{aligned}
$$

where the summation of four residues are

$$
\begin{aligned}
& \frac{1}{21 \sqrt{2} y^{2}} \cdot \frac{\left(c_{1} c_{3}-c_{2} c_{4}\right) \cos t-\left(c_{1} c_{4}+c_{2} c_{3}\right) \sin t}{c_{1}^{2}+c_{2}^{2}} \\
& +\frac{e^{11 t / 3 y^{2}}}{7 \sqrt{455}\left(c_{5}^{2}+c_{6}^{2}\right)} \\
& +\left[-5 \sqrt{70} r^{2.5}\left(-c_{6} \cos \left(2.5 \theta+\frac{\sqrt{455} t}{3 y^{2}}\right)\right.\right. \\
& \left.+c_{5} \sin \left(2.5 \theta+\frac{\sqrt{455} t}{3 y^{2}}\right)\right) \\
& +518 r^{2}\left(-c_{6} \cos \left(2 \theta+\frac{\sqrt{455} t}{3 y^{2}}\right)\right. \\
& \left.+c_{5} \sin \left(2 \theta+\frac{\sqrt{455} t}{3 y^{2}}\right)\right) \\
& \quad 288 \sqrt{70} r^{1.5}\left(-c_{6} \cos \left(1.5 \theta+\frac{\sqrt{455} t}{3 y^{2}}\right)\right.
\end{aligned}
$$



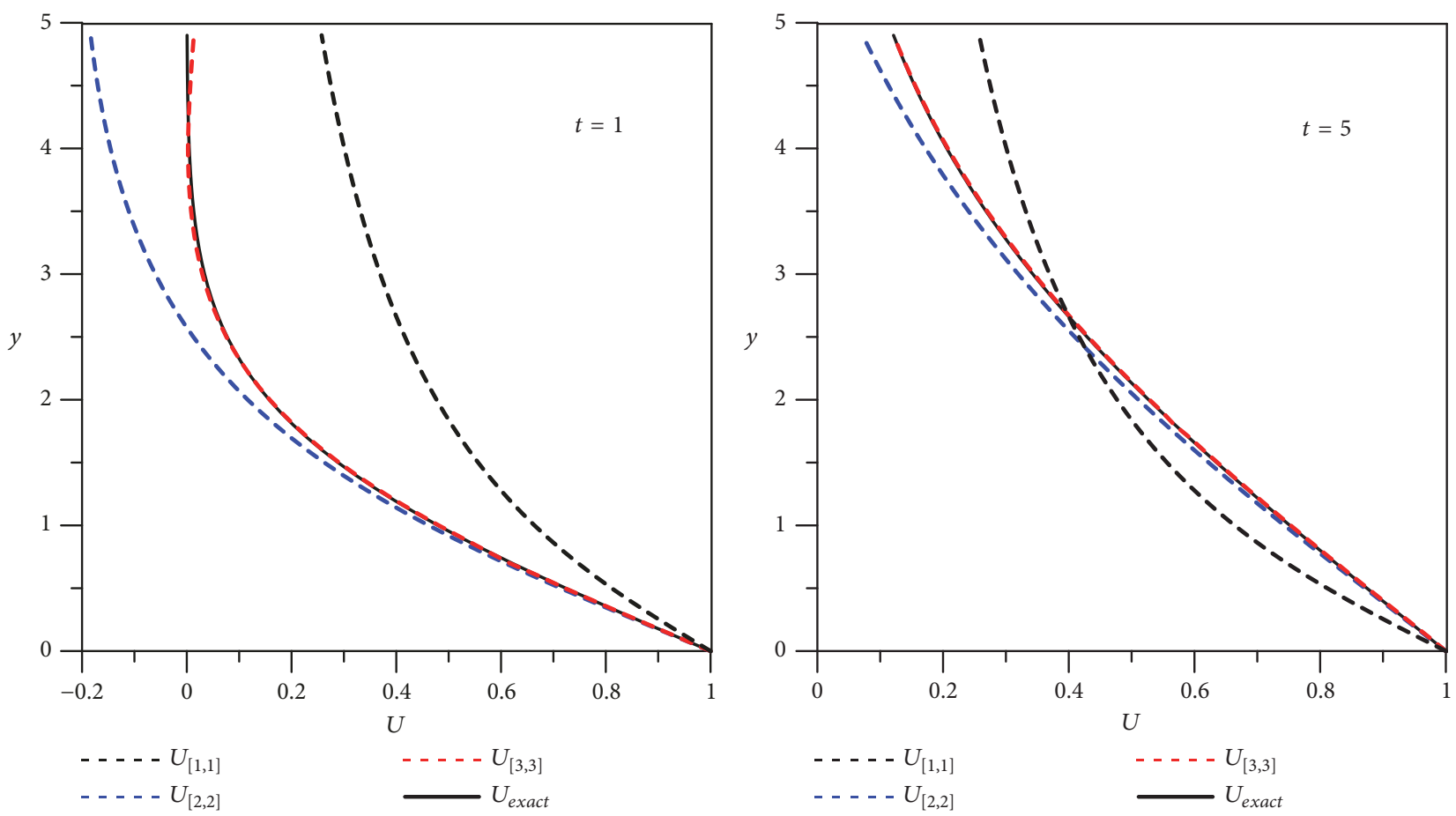

FIGURE 4: Velocity profiles of the exact and ADM-Padé solutions for the unbounded Stokes' first problem. (a) $t=1$. (b) $t=5$.

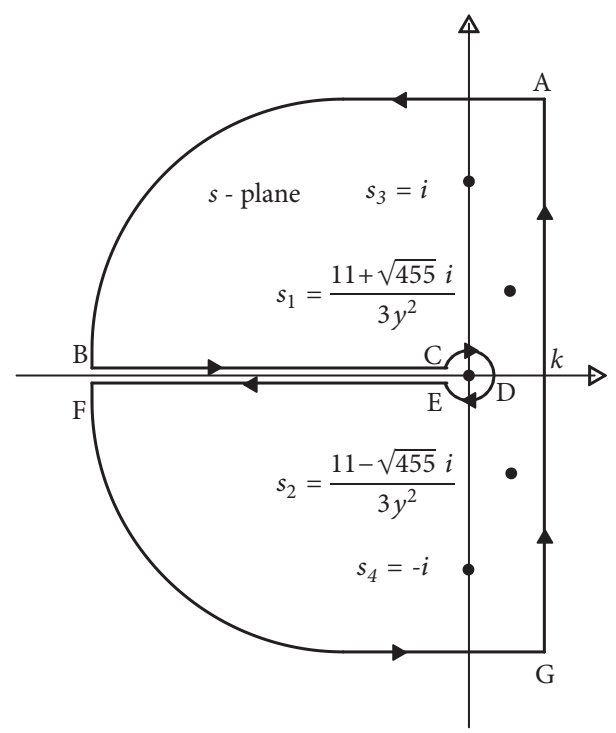

FIGURE 5: Contour integral of unbounded Stokes' second problem.

$$
\begin{aligned}
& \left.+c_{5} \sin \left(1.5 \theta+\frac{\sqrt{455} t}{3 y^{2}}\right)\right) \\
& +4032 r\left(-c_{6} \cos \left(\theta+\frac{\sqrt{455} t}{3 y^{2}}\right)\right. \\
& \left.\left.+c_{5} \sin \left(\theta+\frac{\sqrt{455} t}{3 y^{2}}\right)\right)\right]
\end{aligned}
$$

with $r$ and $\theta$ are defined in (63), and coefficients $c_{1}$ to $c_{6}$ are

$$
\begin{aligned}
& c_{1}=\frac{64}{y^{2}}-1 \\
& c_{2}=\frac{22}{3 y^{2}} \\
& c_{3}=5 \sqrt{210} y-\frac{96 \sqrt{210}}{y}+\frac{1344 \sqrt{2}}{y^{2}}
\end{aligned}
$$



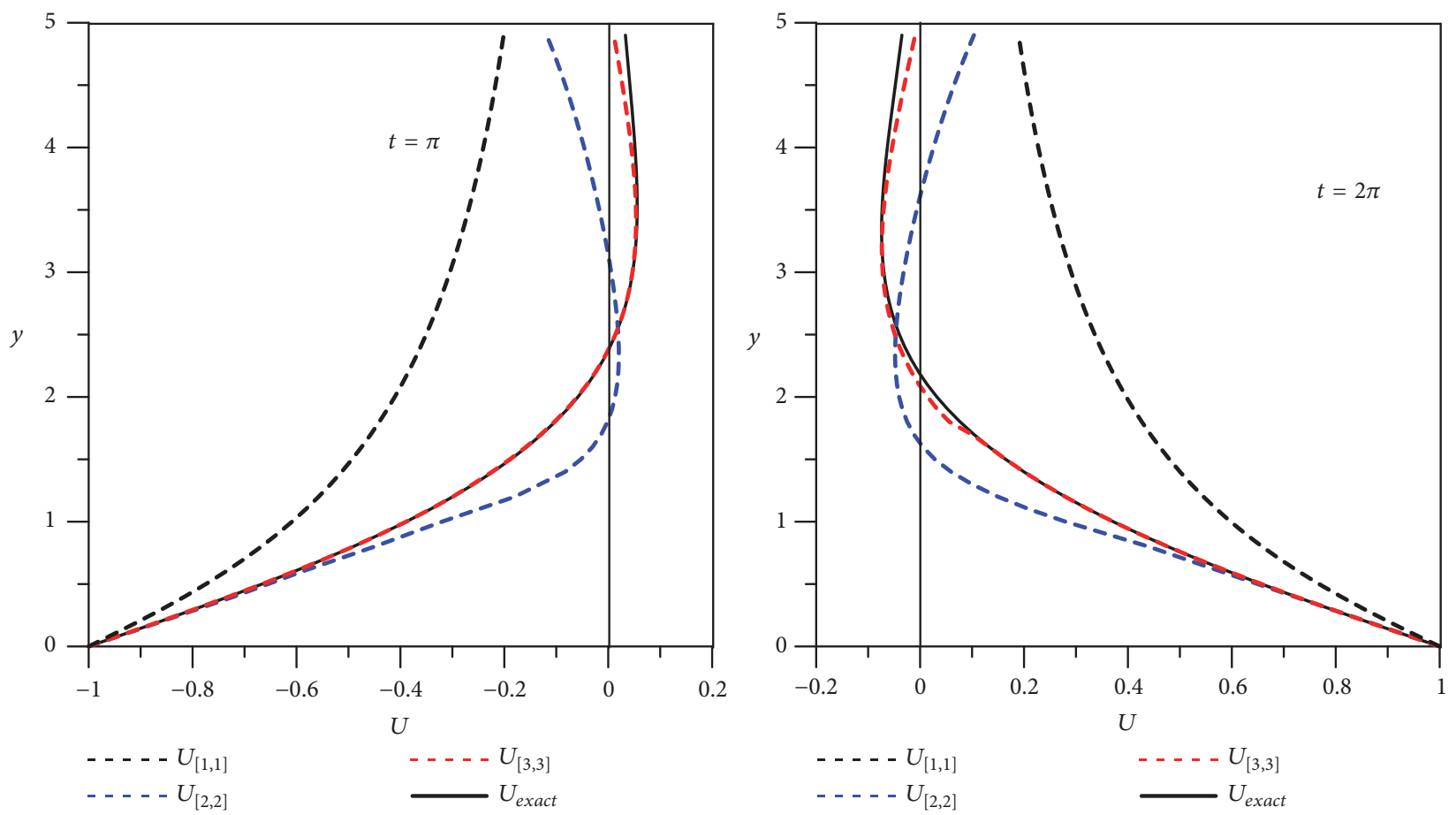

Figure 6: Velocity profiles of the exact and ADM- Padé solutions for the unbounded Stokes' second problem. (a) $t=\pi$. (b) $t=2 \pi$.

$$
\begin{aligned}
& c_{4}=-5 \sqrt{210} y+518 \sqrt{2}-\frac{96 \sqrt{210}}{y} \\
& c_{5}=9 y^{4}-334 \\
& c_{6}=22 \sqrt{455} .
\end{aligned}
$$

The exact solution is also shown for comparison [16]:

$$
\begin{aligned}
& U_{\text {exact }}=\frac{1}{2} \\
& \cdot \operatorname{Re}\left\{\exp \left[\frac{-y}{\sqrt{2}}+i\left(t-\frac{y}{\sqrt{2}}\right)\right] \operatorname{Erfc}\left(\frac{y}{2 \sqrt{t}}-\sqrt{i t}\right)\right. \\
& \left.\quad+\exp \left[\frac{y}{\sqrt{2}}+i\left(t+\frac{y}{\sqrt{2}}\right)\right] \operatorname{Erfc}\left(\frac{y}{2 \sqrt{t}}+\sqrt{i t}\right)\right\}
\end{aligned}
$$

where Re represents the real part of the complex. Figure 6 displays the velocity distributions of the ADM-Padé solutions for $t=\pi$ and $t=2 \pi$ for the range $0 \leq y \leq 5$. Dash and solid curves represented the ADM-Padé solutions and the exact solution, respectively. It is found that the higherorder ADM-Padé solution provides a much better behavior than the lower-order one. Similar to the bounded case, the steady-state and the transient parts can be readily obtained by separating oscillating and nonoscillating terms.

\section{Conclusions}

In this paper, we examined of Stokes' first and second problems by applying the Adomian decomposition method with the help of the Padé approximant. Both bounded and unbounded cases are analyzed to acquire the approximate solutions. Two major concerns including the determination of the unknown in the ADM solution and the treatment of the unbounded domain are demonstrated and solved. Comparisons between the ADM solutions and the exact solution show an excellent agreement which verifies the accuracy and applicability of the ADM for Stokes' problems.

\section{Data Availability}

The data used to support the findings of this study are included within the article.

\section{Conflicts of Interest}

The authors declare that they have no conflicts of interest.

\section{Acknowledgments}

Financial support from the Ministry of Science and Technology of Taiwan with the grants MOST 106-2221-E-270-002MY2, MOST 106-2911-I-006-301, and MOST 107-3113-E-006004-CC2 is acknowledged.

\section{References}

[1] G. Adomian, "A review of the decomposition method in applied mathematics," Journal of Mathematical Analysis and Applications, vol. 135, no. 2, pp. 501-544, 1988. 
[2] G. Adomian, Solving Frontier Problems of Physics: The Decomposition Method, Kluwer Academic, Dordrecht, The Netherlands, 1994.

[3] T. A. Abassy, M. A. El-Tawil, and H. K. Saleh, “The solution of $\mathrm{KdV}$ and $\mathrm{mKdV}$ equations using Adomian Padé approximation," International Journal of Nonlinear Sciences and Numerical Simulation, vol. 5, no. 4, pp. 327-339, 2004.

[4] A. Sadighi and D. D. Ganji, "Analytic treatment of linear and nonlinear Schrödinger equations: a study with homotopyperturbation and Adomian decomposition methods," Physical Review A, vol. 372, no. 4, pp. 465-469, 2008.

[5] S. M. El-Sayed and D. Kaya, "The decomposition method for solving (2+1)-dimensional Boussinesq equation and (3+1)dimensional KP equation," Applied Mathematics and Computation, vol. 157, no. 2, pp. 523-534, 2004.

[6] T. A. Abassy, M. A. El-Tawil, and H. K. Saleh, "The solution of Burgers' and good Boussinesq equations using ADM-Padé technique," Chaos, Solitons \& Fractals, vol. 32, no. 3, pp. 10081026, 2007.

[7] M. Dehghan, A. Hamidi, and M. Shakourifar, "The solution of coupled Burgers' equations using Adomian-Pade technique," Applied Mathematics and Computation, vol. 189, no. 2, pp. 10341047, 2007.

[8] A. H. Bokhari, G. Mohammad, M. T. Mustafa, and F. D. Zaman, "Adomian decomposition method for a nonlinear heat equation with temperature dependent thermal properties," Mathematical Problems in Engineering, vol. 2009, Article ID 926086, 12 pages, 2009.

[9] M. Dehghan, "Application of the Adomian decomposition method for two-dimensional parabolic equation subject to nonstandard boundary specifications," Applied Mathematics and Computation, vol. 157, no. 2, pp. 549-560, 2004.

[10] A.-M. Wazwaz, "The modified decomposition method and Padé approximants for a boundary layer equation in unbounded domain," Applied Mathematics and Computation, vol. 177, no. 2, pp. 737-744, 2006.

[11] R. Rach, J.-S. Duan, and A.-M. Wazwaz, "Solving coupled Lane-Emden boundary value problems in catalytic diffusion reactions by the Adomian decomposition method," Journal of Mathematical Chemistry, vol. 52, no. 1, pp. 255-267, 2014.

[12] P.-Y. Tsai and C.-K. Chen, "A new algorithm on the solutions of forced convective heat transfer in a semi-infinite flat plate," Journal of Mechanics, vol. 27, no. 1, pp. 63-69, 2011.

[13] Y. Zeng, "The Laplace-Adomian-Padé technique for the ENSO model," Mathematical Problems in Engineering, vol. 2013, Article ID 954857, 4 pages, 2013.

[14] S. A. Khuri, "A Laplace decomposition algorithm applied to a class of nonlinear differential equations," Journal of Applied Mathematics, vol. 1, no. 4, pp. 141-155, 2001.

[15] G. G. Stokes, "On the effect of the internal friction of fluids on the motion of pendulums," Transactions of the Cambridge Philosophical Society, vol. 9, pp. 8-106, 1851.

[16] C.-M. Liu and I.-C. Liu, "A note on the transient solution of Stokes' second problem with arbitrary initial phase," Journal of Mechanics, vol. 22, no. 4, pp. 349-354, 2006.

[17] S. P. Hu, C. M. Fan, C. W. Chen, and D. L. Young, "Method of fundamental solutions for Stokes' first and second problems," Journal of Mechanics, vol. 21, no. 1, pp. 25-31, 2005.

[18] C.-M. Liu, "On the study of oscillating viscous flows by using the Adomian-Padé approximation," Journal of Applied Mathematics, vol. 2015, Article ID 864190, 5 pages, 2015.
[19] C.-M. Liu, "Application of the Adomian decomposition method to oscillating viscous flows," Applied and Computational Mathematics, vol. 5, no. 3, pp. 121-132, 2016.

[20] A. M. Siddiqui, M. Hameed, B. M. Siddiqui, and Q. K. Ghori, "Use of Adomian decomposition method in the study of parallel plate flow of a third grade fluid," Communications in Nonlinear Science and Numerical Simulation, vol. 15, no. 9, pp. 2388-2399, 2010.

[21] C.-M. Liu, "Complete solutions to extended Stokes' problems," Mathematical Problems in Engineering, vol. 2008, Article ID 754262, 18 pages, 2008. 


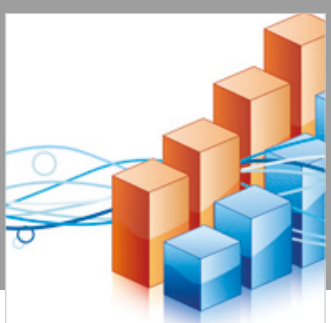

Advances in

Operations Research

\section{-n-m}
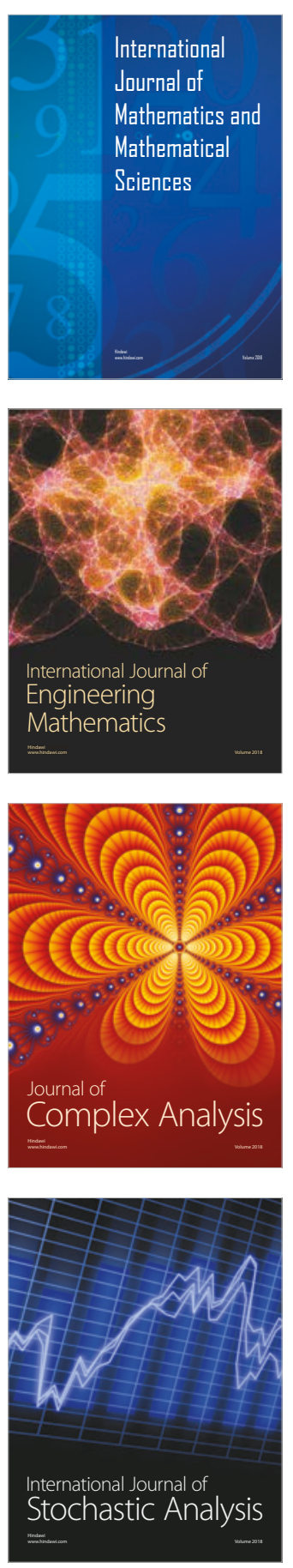
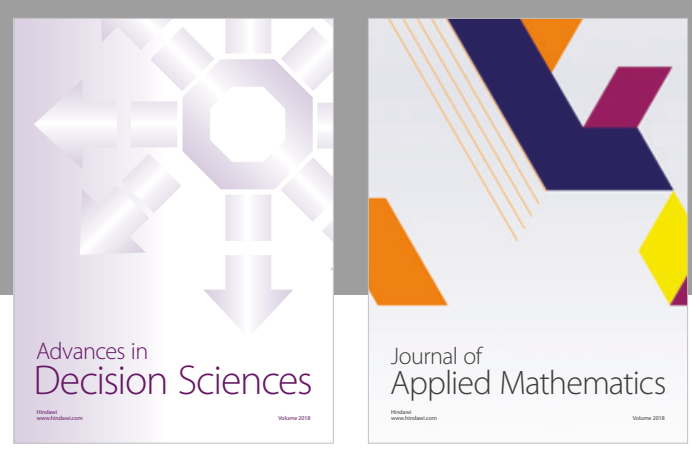

Journal of

Applied Mathematics
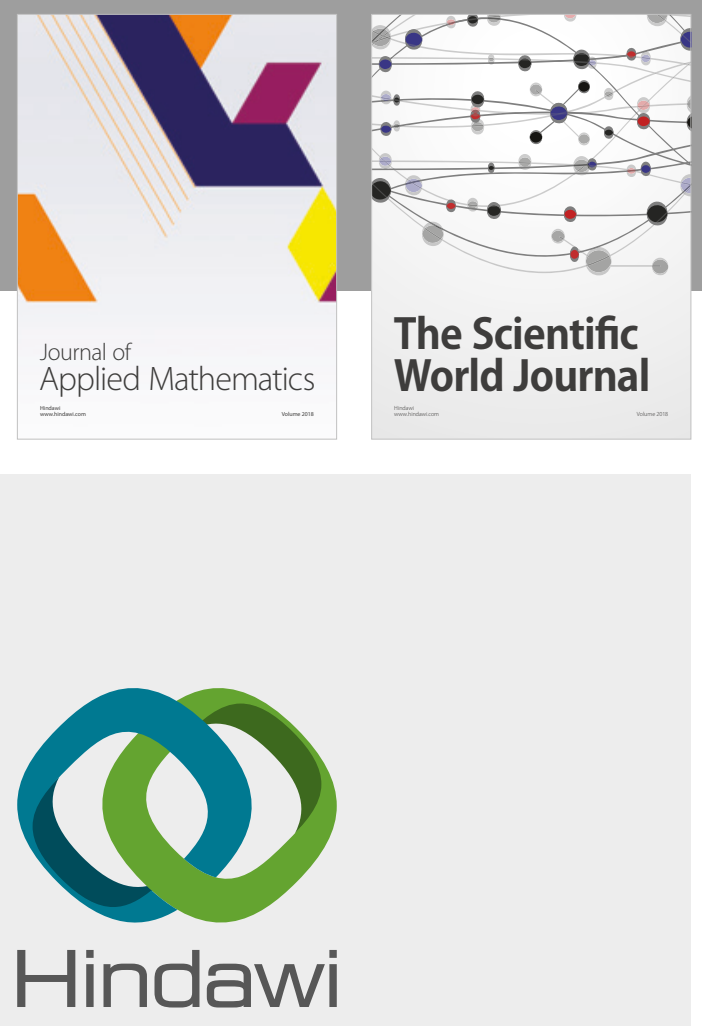

Submit your manuscripts at

www.hindawi.com

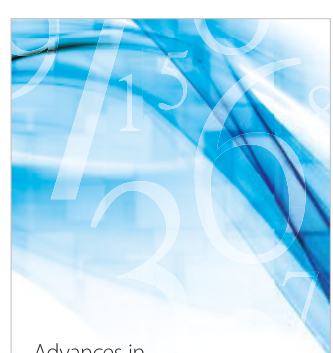

Advances in
Numerical Analysis
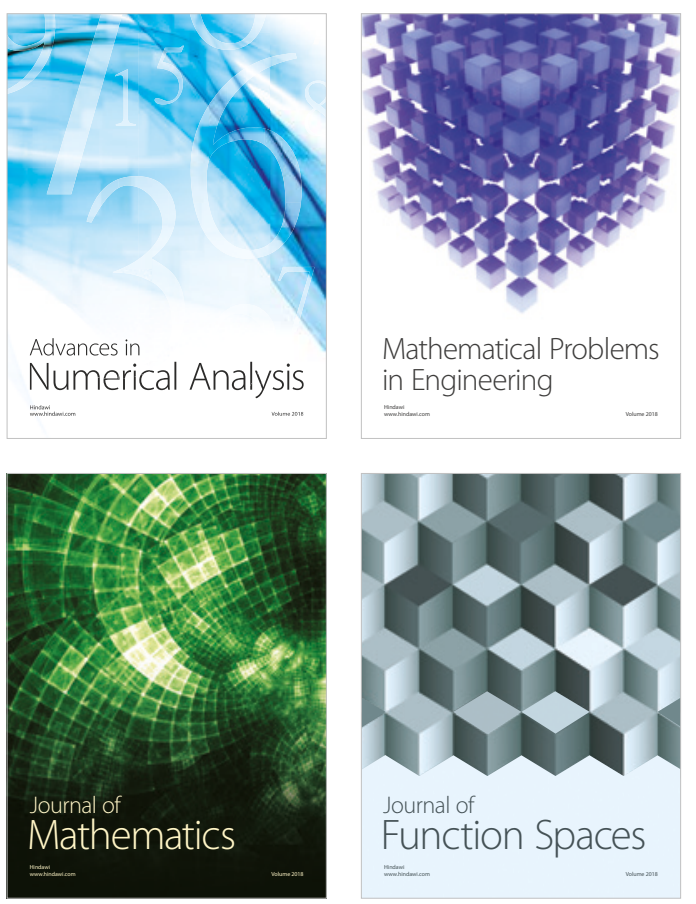

Mathematical Problems in Engineering

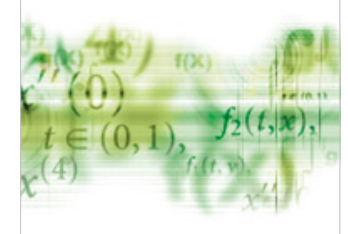

International Journal of

Differential Equations

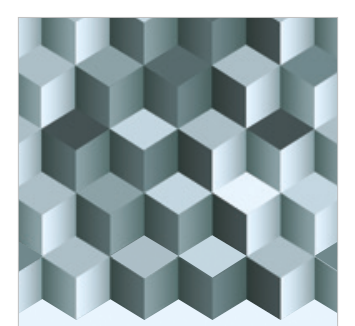

Journal of

Function Spaces

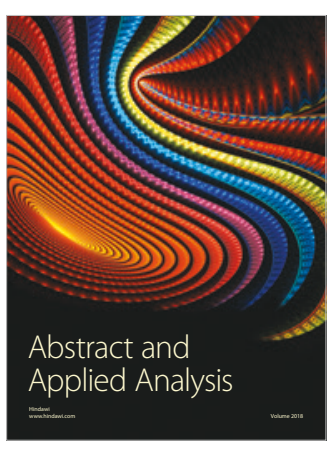

The Scientific

World Journal

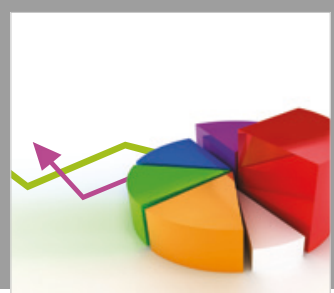

Journal of

Probability and Statistics
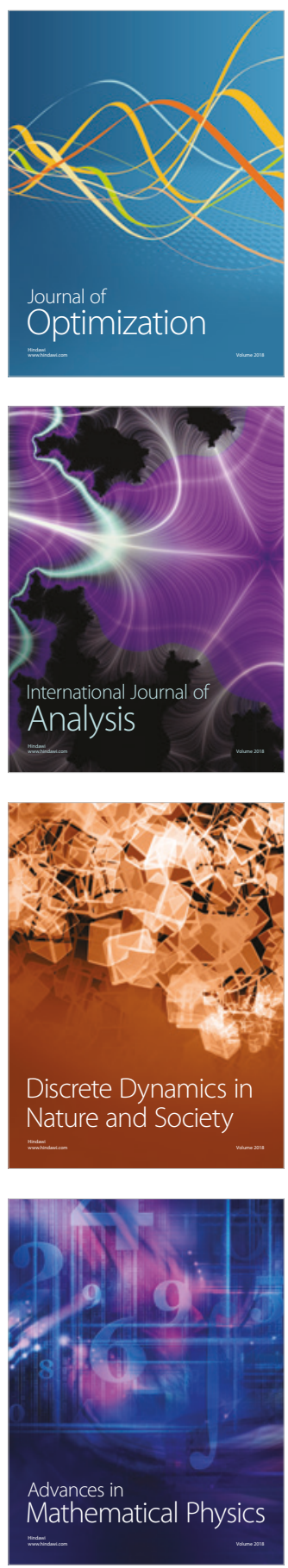